\title{
Screening for retinopathy of prematurity
}

\author{
Alistair R Fielder, Malcolm I Levene
}

The demise of retinopathy of prematurity (ROP) was confidently forecast in the $1950 \mathrm{~s}$ when supplemental oxygen was identified as a causal factor. Unfortunately this did not transpire and by the mid-1960s it became all too apparent that ROP was not solely of historical interest. The purpose of this article is to consider screening for ROP working on the basis that at present this condition is not entirely preventable, and that despite meticulous neonatal care, serious and blinding disease still occurs. ${ }^{1}$ As in the past the ophthalmologist could neither prevent nor treat ROP, effectively all responsibilities for this condition, including its medicolegal aspects, rested with issues related to causation. The results of the Multicenter Trial of Cryotherapy for Retinopathy of Prematurity have changed all that. ${ }^{2-4}$ After more than 20 years of inconclusive studies, retinal ablative treatment has been shown to significantly reduce the unfavourable outcome of severe disease, both in terms of the retinal appearance and visual function. ${ }^{2-4}$ Screening has now become a vital practical issue.

In this article the nature of the preterm population will be briefly considered, after which certain aspects of ROP will be discussed such as: its classification, incidence and severity, natural history, and treatment. Against this background the concepts, and finally, the guidelines for screening will be considered. These were drawn up by a working party convened jointly by the College of Ophthalmologists and the British Association of Perinatal Medicine. ${ }^{5}$ Other than the guidelines themselves, the views expressed in this article are not necessarily those of the working party.

\section{The preterm population}

Over the last 40 years, survival rates for the baby born before term have dramatically increased. In 1988 the mortality rates for infants of birth weight $<1500 \mathrm{~g}$ in England and Wales was $74 \%$ and there has been a very considerable improvement in survival of the tiniest infants within this group. In the 1940 s only about $8 \%$ of neonates $<1000 \mathrm{~g}$ birth weight survived the neonatal period, ${ }^{7}$ but in recent years this figure is in the region of $60 \%$. Concomitant with this improved survival, the incidence of ROP has risen and it is predominantly among the most immature section of the preterm population that the advanced stages of this disease occur.

\section{Classification}

The introduction of an internationally agreed classification of $\operatorname{ROP}^{8} 9$ (table 1) has been one of the most important events in the history of this condition. First, it has made possible direct comparison between centres, and provided the impetus for a wave of research activity. Second, if utilised fully and with an understanding of the natural history of ROP, it serves as a practical guide both to the kinetics of the ROP process and the potential for progression to severe disease. Above all, it is logical and simple to use. Being concerned

\section{Table 1 International classification of ROP}

\section{Severity by stage}

(1) Demarcation line

Thin white line, lying within the plane of the retina and

(2) Ridge

The line of stage 1 has increased in volume to extend out of the plane of the retina.

Isolated vascular tufts may be seen posterior to the ridge at this stage

(3) Ridge with extraretinal fibrovascular proliferation This may be:

(i) Continuous with the posterior edge of the ridge

(ii) Posterior, but disconnected, from the ridge

(iii) Into the vitreous

(4) Retinal detachment-subtotal

(A) Extrafoveal

(B) Involving the fovea

(5) Retinal detachment-total

Funnel:

Anterior Posterior

$\begin{array}{ll}\begin{array}{l}\text { Anterior } \\ \text { open }\end{array} & \begin{array}{l}\text { Posterior } \\ \text { open }\end{array}\end{array}$

narrow narrow

$\begin{array}{ll}\text { open } & \text { narrow } \\ \text { narrow } & \text { open }\end{array}$

'Plus' disease

Signs of activity which can accompany any stage and include: Retinal vessels-tortuosity and engorgement

Iris-vascular engorgement and rigidity

Vitreous-haze

Location by zone

Retinal blood vessels grow out from the optic disc, and the zonal arrangement reflects this pattern of vascular development. Being centred on the fovea, the neural organisation of the retina is eccentric to the vascular pattern.

The zones are centred on the optic disc but zone 3 is crescentic being widest in the temporal retina and absent nasally Zone 1

Extends from the optic disc to twice the disc-foveal distancea radius of $30^{\circ}$

Zone 2

Extends from the periphery of the nasal retina (ora serrata) in a circle around the anatomical equator. In the temporal retina, in the absence of an anatomical landmark zone 3 cannot be identified precisely. Only known to be entered with certainty when the nasal retina is fully vascularised

Zone 3

Anterior to zone 2, is present temporally, inferiorly and superAnterior to zone 2 , is present te
iorly, but not in the nasal retina

iorly, but not in the

Extent of ROP
This is recorded as clock hours in each eye in the appropriate zone 
with the acute process, the classification of regressed ROP $^{9}$ will not be considered here.

This classification is based on three concepts, all fundamental for our understanding of the natural history of ROP, including its potential for progression. First, grading the severity of ROP by stage. Second, the location of the response by retinal zone, and third, its circumferential extent by clock hour on the retina. The relevance of these concepts to the natural history and routine screening of ROP will be considered later.

\section{ROP - incidence and severity}

There has been much talk of the two ROP epidemics. ${ }^{1}$ The first, which commenced in 1942, was brought to an end about a decade later by oxygen restriction. The second epidemic began in the late 1960 s, concomitant with advances in neonatal medicine, and is ongoing. Interestingly over these four decades the risk of ROP induced blindness has remained the same for infants 750-999 $\mathrm{g}$ birth weight (very few of whom survived in the first epidemic), but for those $1000-1500 \mathrm{~g}$ birth weight this risk is now very considerably reduced. 1011 This is evidence that advances in neonatal medicine since the 1950s have reduced the risk of severe ROP in the larger infants $(>1000 \mathrm{~g})$, and brought to an end what could retrospectively be called the preventable epidemic. On the other hand these same advances which terminated the first epidemic have resulted in the increased survival of the very immature neonate and given rise to the second epidemic, which is currently not preventable.

Against this background it is now appropriate to consider current ROP incidence estimates. These vary ${ }^{12-20}$ but the following figures have been published for given birth weights: $<1000 \mathrm{~g}, 53.0 \% \%^{12}$ and $88.5 \%{ }^{18}$; $\leq 1250 \mathrm{~g} ; 42.9 \%^{19}$ and $65.8 \% \%^{20}$; $\leq 1300 \mathrm{~g}, 55 \cdot 6 \% \%^{15}$ and $75 \cdot 4 \%{ }^{18}$; and $\leq 1500 \mathrm{~g}$, $34 \cdot 9 \%, 1348 \cdot 1 \%,{ }^{14}$ and $60 \cdot 1 \% .^{18}$ Thus incidence rises with increasing immaturity and the same can be said for severity, so that by birth weight the incidence of stage three ROP is as follows: $<750 \mathrm{~g}, 37.4-40.0 \%$; $750-1000 \mathrm{~g}, 19 \cdot 0-24 \cdot 7 \%$; and $1000-1250 \mathrm{~g}$, $2 \cdot 7-8 \cdot 5 \%$. ${ }^{18-20}$ A breakdown of results by gestational age is as follows: $\leq 27$ weeks, $25 \cdot 0-28 \cdot 9 \%$; and 28-31 weeks, 2.2$10.9 \% .{ }^{18-20}$ Even given an agreed classification, it is not surprising that estimates vary $^{12-20}$; this is due to variations in survival rate, differing methods of both patient selection and the technique, and frequency of examination. The purpose of this section is not to analyse incidence rates in detail but to show the trend that the incidence and severity of ROP both rise with increasing prematurity so that below about $1300 \mathrm{~g}$ birth weight infants are more likely than not to develop ROP. Severe disease is confined mainly, but not entirely, to those infants of birth weight $<1250 \mathrm{~g}$ and $\leq 30$ weeks' postmenstrual age.*

\section{Natural history}

Appreciation of the natural history forms a logical basis for the design of a screening programme. Here three topics are pertinent: the age at onset of ROP, rate of progression, and retinal location.

\section{(1) AGE AT ONSET}

ROP only affects immature retinal vessels and does not occur therefore after the retina is fully vascularised. Instinctively perhaps one would expect the most premature and often ill neonate, with a very immature retinal vascular system, to develop ROP sooner postnatally than his/her larger more mature and relatively well counterpart. This is not so, as the postnatal age at onset is later for the more immature neonate. However, when corrected for the degree of prematurity, ROP develops over a relatively narrow postmenstrual age range. ${ }^{20-22}$ To quote one study, ${ }^{22}$ the onset of any ROP was between 29.7 and 45.0 weeks' postmenstrual age, with $92 \cdot 1 \%$ between 30.0 and 40.0 weeks, $80.8 \%$ between 30.0 and 37.8 weeks, and $75.0 \%$ between 30.0 and 36.0 weeks. Of the 23 infants who developed ROP after term, all were relatively mature and none went on to severe (stage 3 ) disease. This indicates that ROP onset is linked more to the stage of development of the infant, probably at a retinal level, that neonatal events. However ROP is not present at birth, and this event is necessary for its development. The propensity for severity is governed to a large extent by the state of retinal vascularisation and of course neonatal events. Thus the later the event of birth, the later postmenstrual age at which ROP commences (albeit often shorter postnatal age). ROP commencing at a later postmenstrual age develops in a more vascularised retina and has a low risk for progression to severe disease. ROP commencing after 35 weeks postmenstrual age is most unlikely to reach stage 3 . The suggestion that onset in the most immature neonate may be hastened very slightly does not substantially alter these comments. $^{21-23}$

\section{(2) RATE OF PROGRESSION}

As with the age at onset, the rate of progression is also governed predominantly by the stage of development rather than by postnatal age, time, or severity, of neonatal events such as oxygen treatment or illnesses. ${ }^{20-23}$ It is important to emphasise that the importance of neonatal events on the severity of ROP is not questioned, but here only the timing of ROP onset and progression are being considered. The median postmenstrual age at which the various stages develops is almost identical in two studies 2022 and is as follows by stages: stage $1,34.3$ weeks; stage $2,35 \cdot 4$ weeks; stage 3, 36.6 weeks; and for threshold ROP at 36.9 weeks' postmenstrual age (fig 1$).{ }^{20}$

*Gestational age is the period in utero and is calculated as commencing from the first day of the last menstrual period. Once birth has occurred gestational age is inappropriate, and postnatal age, postmenstrual age, and postconceptional age are postnatal age, postmenstrual age, and postconceptional age are used. Postmenstrual age is preferred to denote gestational age plus postnatal age, in weeks. Postconceptional age is 


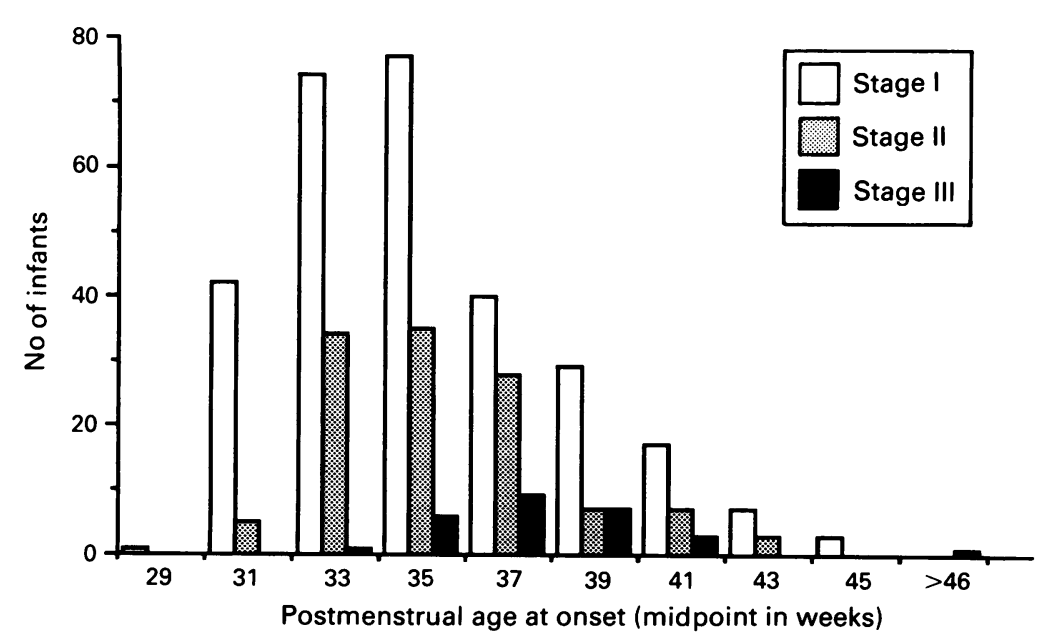

Figure 1 Postmenstrual age at development of ROP stages 1, 2, and 3 in 572 neonates. ${ }^{22}$ No ROP developed until the 30th week, although 201 were $\leq 29$ weeks' gestational age. Both onset and progression on largely determined by postmenstrual age. The one infant who developed stage 3 at $>46$ weeks had not been examined for four weeks previously. The priority of screening is to identify infants with, or who are at risk of developing, stage 3 ROP.

(3) RETINAL LOCATION

The more premature the neonate, the less advanced retinal vascular development, the more posterior by zone the location of the retinopathy, and the greater the propensity for progression. It follows that zone 1 disease has a high tendency to progress to stage 3 , but for ROP confined entirely to zone 3 this rarely if ever happens.

Traditional teaching has it that ROP commences in the temporal retina as this is the last region to vascularise. Recently it has been observed that in the most immature neonate ROP commences preferentially in the nasal retina and later extends to other regions. ${ }^{22} 24$ Both inferior and superior retinal regions are less frequently involved at onset unless the process is located posteriorly and liable therefore to become severe. The finding of ROP in these regions early in the course of the disease is a useful indicator of the possibility of future severity (figs 2 and 3 ).

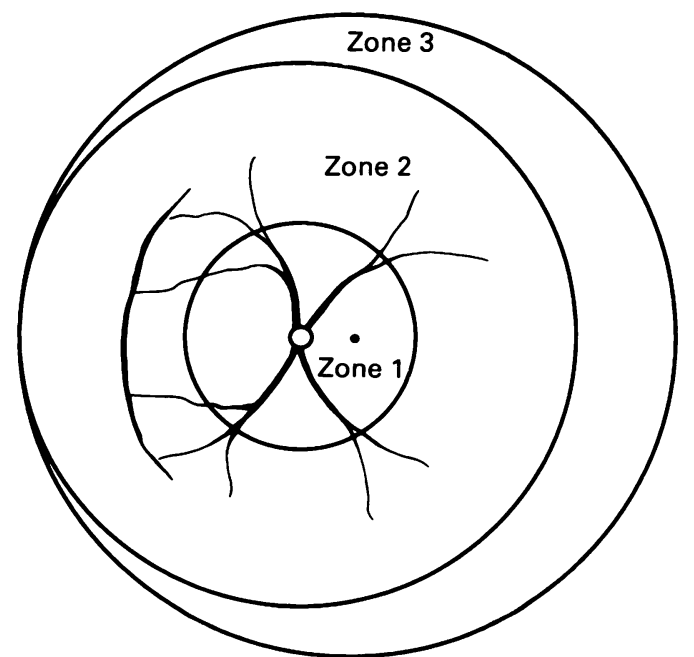

Figure 2 Stage 1 ROP in the nasal retina. This is a frequent site of initial involvement in the most immature neonate and carries a high risk for extension, by clock hour and progression to stage 2 or 3. Central black spot denotes the macula.

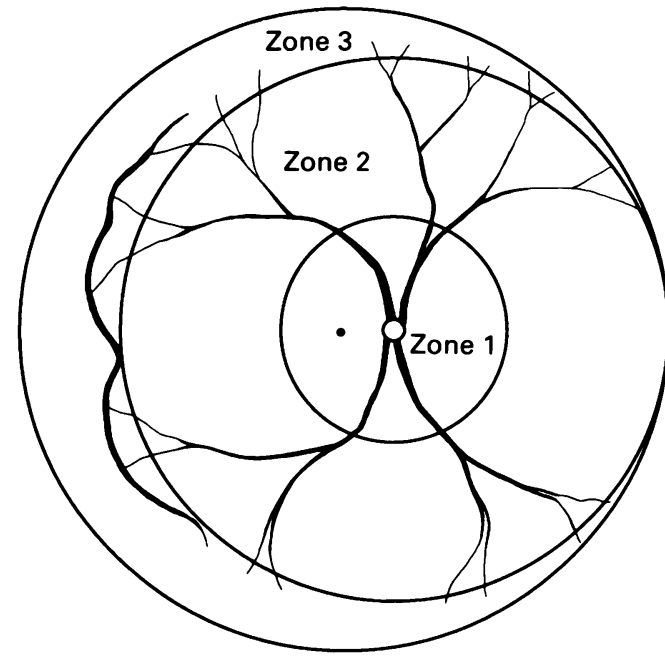

Figure 3 Stage 2 ROP confined to the temporal retina. Located in zone 3, the risk for progression to threshold disease is so low that in clinical terms it can be ignored. Central black spot denotes the macula.

\section{OTHER ASPECTS}

In contrast to cicatricial ROP, which is characteristically asymmetrical, acute ROP tends to be symmetrical by stage (rarely exceeding more than one stage), although the extent of clock hour involvement can differ between the two eyes. Plus disease is a sign of activity which can be superimposed onto any stage and in practice indicates the eye likely to progress to stage 3 . The most consistent signs of plus disease are dilatation and tortuosity of the posterior retinal vessels, with engorgement of the iris vessels, and vitreous haze in more severe cases.

\section{OUTCOME OF ACUTE ROP}

All highest stage 1 and 2 acute ROP undergo spontaneous resolution. Stage 3 acute ROP may, or may not resolve, depending on its severity, so that when threshold stage (table 2) is reached, full resolution is unlikely and the risk of blindness is predicted to be close to $50 \% .^{2}$

\section{SUMMARY}

Both the incidence and severity of ROP rise with increasing immaturity. Although ROP is a condition of immature retinal vessels, its onset and progression rate are determined predominantly by the stage of development rather than neonatal events. While puzzling from the point of view of basic mechanisms, this pattern simplifies screening programme design. Similarly, the location and the extent of the ROP response can also provide important predictive clues of progression or resolution.

\section{Treatment}

The treatment of ROP falls into two groups:

Table 2 Prethreshold and threshold ROP

Prethreshold ROP.

Zone 1-Any stage

Threshold ROP:

Stage 3-five continuous or eight cumulative clock hours with 'plus' disease 
first, of the acute lesion, and second, for the late vitreoretinal complications. To take the second group first, while vitreoretinal surgery can offer surgical realignment in a proportion of cases, the visual results to date are dismal as very few attain even the lowest measurable acuity. ${ }^{23}$

The principle of treatment for the acute lesion is retinal ablation. A series of clinical studies started by the Japanese in the mid1960s utilised xenon arc and laser photocoagulation, and cryotherapy to achieve this effect. ${ }^{25-28}$ The results of these early studies were inconclusive due to problems of classification, insufficient patient numbers, and a paucity of detailed clinical data. These problems were surmounted by the introduction of the new international classification ${ }^{89}$ and the mounting of a multicentre trial of cryotherapy.

\section{MULTICENTRE TRIAL OF CRYOTHERAPY FOR}

RETINOPATHY OF PREMATURTTY

The primary aim of this randomised study was to determine prospectively whether cryotherapy is effective in the treatment of severe acute ROP. The secondary aim was to study its natural history. Study design will not be considered. ${ }^{2}$ 25-28 Broadly, 9751 infants $\leq 1250 \mathrm{~g}$ birth weight without congenital anomalies were enrolled between 1 January 1986 and 30 November 1987 in 23 centres in the USA. Ophthalmic examinations commenced at six weeks postnatally and were continued at intervals of two weeks until vascularisation was complete. If prethreshold ROP (table 2) was reached the frequency of examination was increased to weekly, and at threshold (table 2 and fig 4) infants were randomly allocated to either treatment (cryotherapy) or control groups, and cryotherapy was then performed within 72 hours.

The preliminary results, ${ }^{2}$ and those obtained at $3^{3}$ and 12 months ${ }^{4}$ corrected age all confirmed a significant reduction in the unfavourable outcome of threshold ROP by just under $50 \%$, as judged by photographs and clinical examination. Indeed, because of these results the trial was terminated early due to the committee's concern for the welfare of neonates at risk who might not receive treatment. Not all threshold ROP behaved identically: symmetrical disease and that located in zone 2 had a significantly better outcome than zone 1 ROP. In addition to anatomical evidence, at 1 year treated infants had significantly better visual acuity (assessed using the acuity card procedure) than controls. However, the seriousness of ROP is highlighted, as $50.6 \%$ controls and $31.9 \%$ of treated infants fell into the low vision or blind category. ${ }^{4}$

\section{SUMMARY}

About two decades after first being mooted, retinal ablative treatment was found to have a significant beneficial effect on the retina, the eye as a whole, and most important on visual functions.

\section{The cryotherapy procedure}

Here only cryotherapy will be considered (reviews ${ }^{27}{ }^{28}$ ), but it has recently been reported that the same effect can be achieved by laser techniques. ${ }^{29} 30$ The introduction of portable instruments which enable laser to be delivered through an indirect ophthalmoscope hold promise for the future.

\section{INDICATIONS}

The indication for cryotherapy is threshold ROP (table 2). The term prethreshold simply alerts the clinician to the possibility of impending treatable disease threshold.

\section{TIMING OF INTERVENTION}

Once threshold ROP has been identified, delay should be kept to a minimum as the time window for treatment is short: two to three weeks at the most. ROP progression is 'locked in' to the postmenstrual age of the baby and threshold develops at a mean postmenstrual age of 37.7 weeks (range 31.9 to
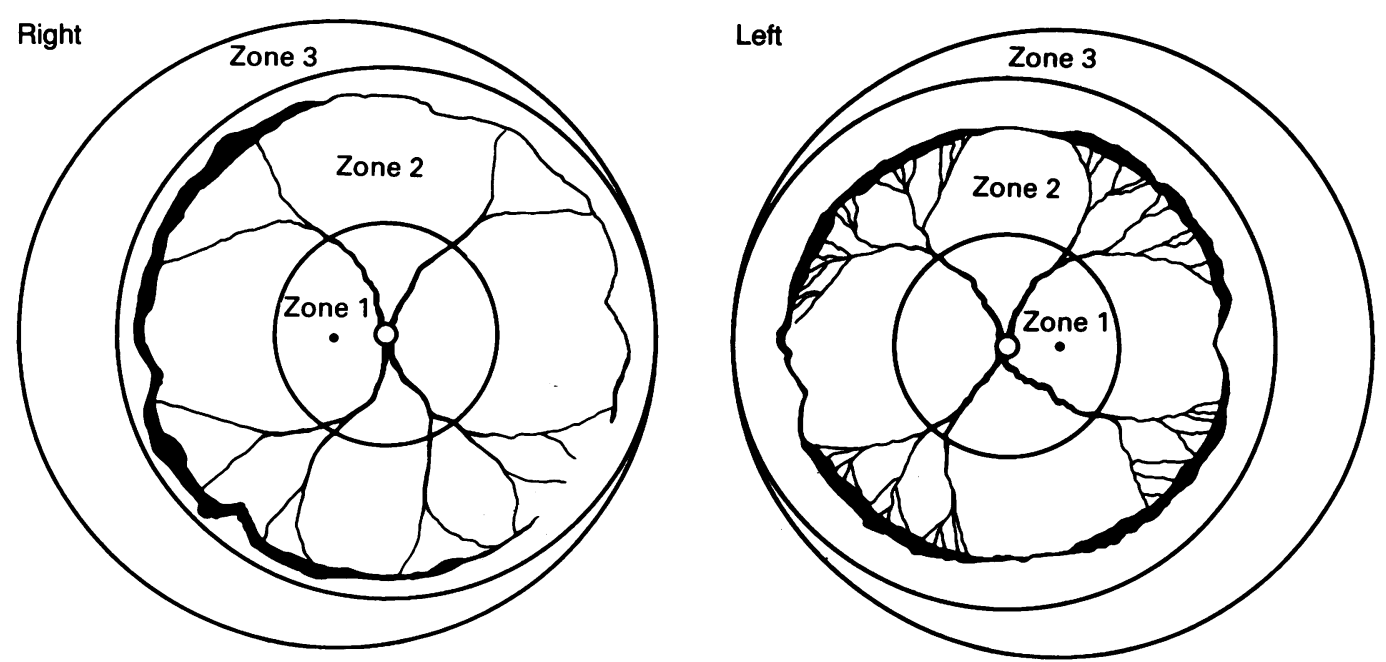

Figure 4 'Threshold' ROP in both eyes. The right eye has 5 continuous clock hours stage 3 ROP. The left eye has $>8$ clock hours stage 3 in total (cumulative). Note the vascular tortuosity which is not present in figs 2 and 3 . This is the most frequently seen sign of 'plus' disease. If the pupil is poorly dilated, but vascular tortosity is present, the retinal periphery must be examined as this eye may be harbouring severe ROP. Central black spot denotes the macula. 
50.5 weeks). ${ }^{320}$ The postnatal age range is 6.6 to 23.9 weeks (mean 11.3 weeks) indicating the value of using postmenstrual age as the guide. $^{320}$

THE INFANT AND FAMIII

It hardly needs to be stated that the infant requiring cryotherapy is immature and fragile. Clearly the general wellbeing of the baby must be the first consideration, but the narrow time available for treatment must not be overlooked. This is also a difficult time for parents, who perhaps are just beginning to relax about their infant's general condition. Imparting the news that their baby has a potentially blinding condition obviously requires the utmost diplomacy.

\section{ANAESTHESIA}

Cryotherapy is painful and the procedure should be performed under either general anaesthesia or local anaesthesia with sedation. More important than the type of anaesthesia is the involvement of a neonatatologist or an experienced paediatric anaesthetist.

For local anaesthesia, the procedure is performed on the neonatal unit and the infant should remain in hospital overnight. Topical anaesthetics should be in eyedrop form, rather than subconjunctival and retrobulbar routes, which carry unacceptable risks. ${ }^{31}$ Resuscitation equipment must be to hand, and an intravenous line in place for the administration of sedation or resuscitation agents before starting the procedure. Systemic complications have been reported and these include bradycardia, cyanosis, and respiratory depression, ${ }^{31}$ but no fatalities.

\section{CRYOAPPLICATION}

After dilatation of the pupil, the cryotherapy procedure is performed under direct visualisation through the indirect ophthalmoscope (fig 5). An eyelid speculum opens the eyelids and cryotherapy is applied using a curved metallic probe of about $2-3 \mathrm{~mm}$ diameter that is rounded at the tip (a variety of probe designs exist). Without opening the con-

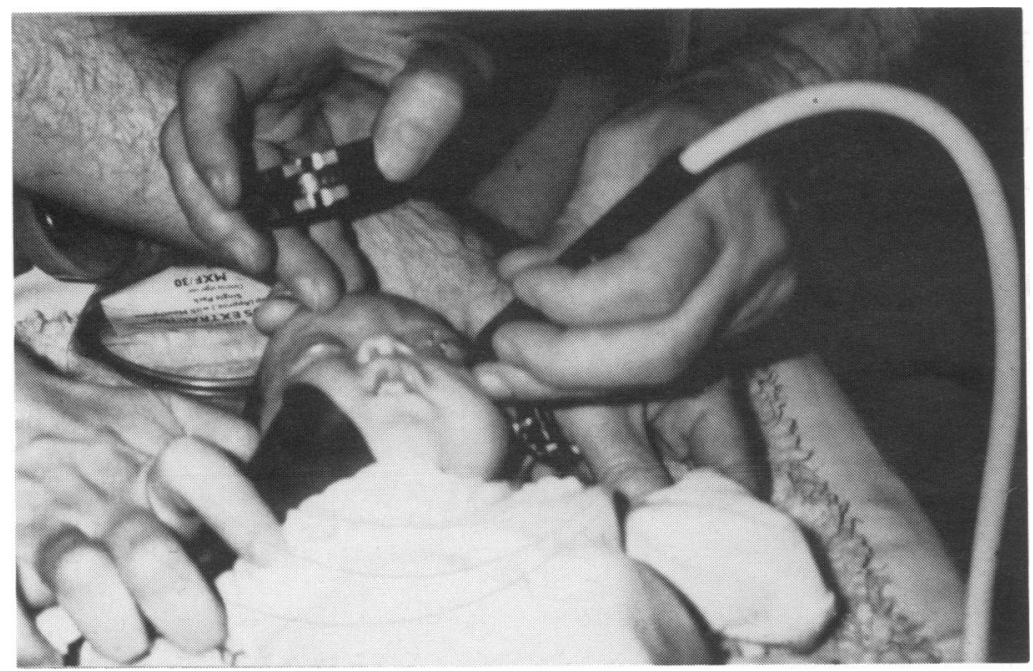

Figure 5 Cryotherapy being performed under sedation and with topical anaesthesia. The cryoprobe is placed against the sclera. Reproduced with permission from Recent Advances in Ophthalmology 8; SD Davidson, B fay, editors. Churchill Livingstone, $1992 .{ }^{28}$ junctiva (this may be necessary occasionally) the probe is placed against the globe and freezes rapidly to about $-60^{\circ} \mathrm{C}$. The aim of treatment is to produce a series of lesions covering the entire avascular retina, anterior to the retinopathy. The cryotherapy probe is applied to the globe until the white of the freeze is just visible ophthalmoscopically. When each application is completed the probe reheats and is moved to an adjacent area. It is important to emphasise that it is not the lesion itself that is treated but the avascular retina anterior to this. Regardless of the site of the ROP, current protocols indicate that the entire $360^{\circ}$ globe circumference is treated and this usually requires two rows of cryotherapy.

\section{POSTOPERATIVE MANAGEMENT}

Apart from the instillation of topical mydriatic (not atropine) for a few days, no other medication is required. Within $24-72$ hours the signs of 'plus' disease begin to subside.

Retreatment may be necessary if 'plus' signs fail to subside and if it becomes apparent that certain areas of the eye have not been treated adequately: that is failure for pigmented lesions to appear in about five to seven days. Retreatment should be undertaken within two to three weeks.

\section{COMPLICATIONS}

Conjunctival lacerations and haemorrhages can occur, as can preretinal and vitreous haemorrhage. ${ }^{328}$ Generally these do not cause long term adverse consequences.

\section{Screening for ROP}

The ophthalmologists' role in screening has always been less than satisfactory. In the 1960s it was suggested ROP might be prevented by the ophthalmoscopic monitoring of retinal vessel calibre, ${ }^{32}$ but was soon found not to be feasible. ${ }^{33}$ Furthermore, it soon became apparent that serious ROP occurred despite the most meticulous neonatal care. As ROP could be neither prevented nor effectively treated, screening was not a high priority. This all changed in 1988 when the Multicenter Trial of Cryotherapy for Retinopathy of Prematurity reported that the unfavourable outcome in severe ROP can be significantly reduced. ${ }^{2-4}$ Screening is now an important clinical issue and has been the subject of a number of publications. ${ }^{54-41}$

\section{PURPOSE OF SCREENING}

To identify, with the minimal use of resources: (1) severe (stage 3) ROP requiring treatment-'threshold' disease and (2) ROP with the potential to reach 'threshold'. Pertinent for infants to be transferred to another neonatal unit, or to home, before the time for stage 3 ROP.

PRINCIPLES OF SCREENING PROGRAMME DESIGN Ophthalmic services in the UK, and presumably in many other countries, are severely stretched. Thus any screening programme should not take up an inappropriate amount 
of the available resources. With this in mind, and an understanding of the natural history of ROP, the time devoted to ophthalmic surveillance can be kept to a minimum. Hopefully most infants will require only one assessment.

For the remainder of this article the major issues will be highlighted utilising information discussed previously. To facilitate the reader this will be in note form.

\section{Population at risk}

Severe ROP is confined as far as can be clinically defined to infants $<1500 \mathrm{~g}$ birth weight and $\leq 31$ weeks' gestational age. In the UK there are about 4900 survivors (75\%) of the 6600 infants of $<1500 \mathrm{~g}$ birth weight born in the UK each year.

\section{Timing of ophthalmic examinations}

Principles: (1) onset and progression of ROP are both largely determined by postmenstrual age rather than by neonatal events, thus (2) this parameter (postmenstrual age) predetermines the time of screening examinations. Additional points:

- The time available for treatment is narrow and the examination must be timed accordingly.

-ROP onset is rare before 30 weeks' postmenstrual age.

-A normal examination undertaken very early on (see above) may have no screening value.

-The mean age for the onset of threshold ROP is $37 \cdot 7$ weeks' postmenstrual age. ${ }^{3} 20$ The earliest reported is 31.0 weeks' postmenstrual age, ${ }^{20}$ with 5 th and 95 th centiles of 33.6 and 42.0 weeks' postmenstrual age respectively.

-A first examination after discharge from the neonatal unit may be too late for treatment.

-The assessment of the very sick infant may be deferred for a time, but even so, be aware of the time scale for developing serious ROP.

-Zone 1 and 2 disease should be kept under observation until resolution is well under way.

-The onset of ROP is not related to the timing of oxygen treatment or other neonatal events.

-For those without ROP, once the vessels have entered zone 3, the risk of serious stage 3 has passed and screening may cease (see below).

-For screening purposes it is not necessary to follow up all stages 1 and 2 until the point of complete resolution, it is adequate to ensure that this process is well under way.

\section{ROP location}

Principles: (1) the more posterior the location the greater the propensity for progression to severe ROP and (2) site of onset and involvement can be a useful indicator of subsequent progress - to worsen or resolve.

Additional points:

-Zone 1 and 2 ROP may progress to stage 3.

-ROP confined to zone 3 alone rarely, if ever, progresses to stage 3 .
- ROP is the most immature neonate often starts in the nasal retina.

- Retinopathy involving the nasal retina (that is, zone 2 disease), and/or the vertical regions all have a greater propensity to progress to stage 3 than ROP confined to the temporal retina alone.

- The examiner can only be certain that ROP is located in zone 3 if the nasal retina is fully vascularised (see examination technique later).

Other aspects

Signs of 'plus' disease: pupillary vascular engorgement, pupil rigidity, and engorgement of the posterior pole vessels are all ominous signs - the pupil which fails to dilate to mydriatics may be the one harbouring serious ROP.

\section{Guidelines for ROP screening}

These were published by a working party convened jointly by the College of Ophthalmologists and the British Association of Perinatal Medicine (list of members at end of article). ${ }^{5} \mathrm{~A}$ few aspects of the guidelines as originally published are expanded by the authors of this article.

\section{WHICH BABIES}

(1) $<1500 \mathrm{~g}$.

(2) $\leq 31$ weeks' gestational age.

(3) No sickness criteria. The screening of larger babies who are either very ill or requiring prolonged oxygen treatment has been suggested. The working party did not consider this strictly necessary but, as such infants remain in the neonatal unit for a long time, covering the period at risk, screening of these babies would not pose a significant additional load.

The possibility of visually significant ROP developing in an infant not falling within these criteria cannot be totally excluded

\section{EXAMINATION PROTOCOL:}

(A) Infants $\leq 25$ weeks' gestational age.

(1) 6-7* weeks postnatally, and then (2) every two weeks until 36 weeks' postmenstrual age or until vascularisation is into zone 3 .

(B) Infants 26- $\leq 31$ weeks' gestational age.

(1) 6-7* weeks postnatally, and then (2) 36 weeks' postmenstrual age, or within a week or two of this age, if to be transferred to another hospital, or to be discharged to home around this time.

For many in this group, examinations 1 and 2 will be close, or actually coincide, and one examination will suffice. It makes sense to include at least one examination before discharge from hospital. The clinician needs to understand the rationale for this protocol, and can make the following possible decisions depending on the findings at this time (34-37 weeks' postmenstrual age):

(i) No ROP, and vascularisation is well into zone 3-no review necessary for two reasons:

*In the original publication of the working party, 7 weeks was the recommended age at the first examination. Here we have taken the liberty of broadening this to 6-7 weeks. 
(a) Were severe ROP to develop, by this age (postmenstrual age) the early signs of ROP would already be present and (b) stage 3 is almost never confined to zone 3 .

(ii) ROP in zone 3 and regressing-no review necessary.

(iii) ROP, stage 1 or 2 , but it cannot be determined whether regression has commenced-review in two weeks.

(C) Babies due for transfer to another hospital or for discharge to home.

(1) Before the age of entry into the screening programme. Screening to be undertaken at the receiving hospital - staff should be alerted. If discharged to home the infant should be reviewed at the appropriate time. (2) During the screening period. Infants with ROP which has the potential to progress must be examined at the appropriate times. The only time available for treatment can easily be lost: simple mechanisms need to be established to facilitate review or to alert the staff at the receiving unit.

(D) Follow up.

As dictated by clinical criteria on an individual basis.

The long term review of infants born prematurely, for strabismus, amblyopia, and other visual pathway defects, is beyond the scope of these recommendations, but the review of stage 3 infants is advisable as the incidence of strabismus and other problems is particularly high in this group.

\section{WHO SHOULD EXAMINE?}

Examining preterm neonates demands a high level of ophthalmic expertise and cannot be allocated to the neonatologist or the most junior ophthalmic staff.

\section{EXAMINATION TECHNIQUE}

The following are recommended:

(1) Pupil dilatation by either: (A) guttae cyclopentolate $0.5 \%$ or (B) guttae cyclopentolate $0.5 \%$ with guttae phenylephrine $2.5 \%$. As this dilatation may last up to 26 hours weaker solutions may be employed such as cyclopentolate $0.2 \%$ in combination with phenylephrine $1.0 \%$. All mydriatic eyedrops should be instilled at least 30 minutes before examination.

(2) Indirect ophthalmoscopy with 20 or 28 dioptre lens.

(3) The International Classification for Retinopathy of Prematurity ${ }^{89}$ should be used to record clinical findings.

For the routine screening for severe disease, the use of an eyelid speculum and scleral indentor is not considered mandatory. Nevertheless, they are widely utilised $d^{2-4} \quad 18 \quad 22 \quad 35 \quad 36$ and it is recognised that these instruments do permit the evaluation of the most peripheral regions of the retina, important in determining whether the nasal retina is fully vascularised (that is differentiating zone 2 and zone 3), and accurately defining location. They also facilitate examination when the pupil dilates poorly as in 'plus' disease.
Preferably, topical anaesthetic eyedrops are instilled just prior to using eyelid speculum and scleral indentor.

\section{Conclusions}

In this article we have reviewed the nature of the preterm population and have utilised the natural history of ROP as a basis for screening protocol design for this potentially blinding condition. The reader may be concerned that the criteria recommended for the neonatal period and particularly for follow up are less rigorous that is ideal. This is recognised, but ophthalmic services are stretched, and the needs of the preterm population have to be considered in this wider context. Finally, there is more to prematurity and the visual system than ROP, and hopefully as we gain more knowledge about the nature and mechanisms of these associations, these guidelines will be modified.

ROP screening working party members: Professor Alistair Fielder, College of Ophthalmologists (co-chairman); Professo Alec Garner, College of Ophthalmologists; Mr Stewart Johnston, College of Ophthalmologists; Professor Malcolm Levene, British Association for Perinatal Medicine (co-chairman); Dr Janet Rennie, British Association for Perinata Medicine; Mr Ed Schulenberg, College of Ophthalmologists.

1 Anonymous. Retinopathy of prematurity [Editorial]. Lance 1991;337:83-4.

2 Cryotherapy for Retinopathy of Prematurity Cooperative Group. Multicenter trial of cryotherapy for retinopathy of prematurity: preliminary results. Arch Ophthalmo 1988;106:471-9.

3 Cryotherapy for retinopathy of prematurity: three-month outcome. Arch Ophthalmol 1990;108:195-204

4 Cryotherapy for Retinopathy of Prematurity Cooperative Group. Multicenter trial of cryotherapy for retinopathy of prematurity: one year outcome. Arch Ophthalmo 1990;108:1408-16.

5 Anonymous. College News: ROP screening duty. Quarterly Bulletin of the College of Ophthalmologists 1990; (autumn)

6 Office of Population Census and Surveys. OPCS monitor. Mortality statistics series DH3. London: Office Population Censuses and Surveys, 1988.

7 Silverman WA. Retrolental fibroplasia: a modern parable. New York: Grune and Stratton, 1980.

8 Committee for the Classification of Retinopathy of Prematurity. An international classification of retinopathy Prematurity. An international classification of retinc

9 Committee for the Classification of Retinopathy of Prematurity. An international classification of retinopathy of prematurity. II The classification of retinal detachment. Arch Ophthalmol 1987;105:906-12.

10 Gibson DL, Sheps SB, Schechter MT, Wiggins S, McCormick AQ. Retinopathy of prematurity: a new epidemic? Pediatrics 1989;83:486-92.

11 Gibson DL, Sheps SB, Uh SH, Schechter MT McCormick AQ. Retinopathy of prematurity-induced blindness: birth weight-specific survival and the new epidemic. Pediatrics 1990;86:405-12.

12 Keith CG, Kitchen WH. Retinopathy of prematurity in extremely low birthweight infants. Med $f$ Aust 1984;141:225-7.

13 Reisner SH, Amir J, Shohat M, Krikler R, Nissenkorn Ben-Sira I. Retinopathy of prematurity: incidence and treatment. Arch Dis Child 1985;60:698-701.

14 Schaffer DB, Johnson L, Quinn GE, Weston M, Bowen FW. Vitamin $E$ and retinopathy of prematurity. FW. Vitamin E and retinopathalmology 1985;92:1005-11.

15 Flynn JT, Bancalari E, Bachynski BN, et al. Retinopathy of prematurity. Diagnosis, severity and natural history. prematurity. Diagnosis, severity

16 Schulenberg WE, Prendiville A, Ohri R. Natural history of retinopathy of prematurity. $\mathrm{Br} \mathcal{F}$ Ophthalmol 1987;71: 837-43.

17 Darlow BA. Incidence of retinopathy of prematurity. Arch Dis Child 1988;63:1083-6.

$18 \mathrm{Ng}$ YK, Fielder AR, Shaw DE, Levene MI. Epidemiology of retinopathy of prematurity. Lancet 1988;ii: 1235-8.

19 Acheson JF, Schulenberg WE. Surveillance for retinopath of prematurity in practice: experience from one neonatal intensive care unit. Eye 1991;5:80-5.

20 Palmer EA, Flynn JT, Hardy RJ, et al. The Cryotherapy for Retinopathy of Prematurity Cooperative Group. Incidence and early course of retinopathy of prematurity. Ophthalmology 1991;98:1628-40.

21 Fielder AR, Ng YK, Levene MI. Retinopathy of prematurity: age at onset. Arch Dis Child 1986;61:774-8. 
22 Fielder AR, Shaw DE, Robinson J, Ng YK. Natural history of retinopathy of prematurity: a prospective study. Eye (in press).

23 Quinn GE, Johnson L Abbasi S. Onset of retinopathy of prematurity as related to postnatal and postconceptual prematurity as related to pos:284-8.

24 Fielder AR, $\mathrm{Ng} \mathrm{YK}$, Levene MI, Shaw DE. Retinopathy of prematurity: age at onset and the initial site of involvement. A preliminary report. In: Ben Ezra D, Ryan SJ, ment. A preliminary report. In: Ben Ezra D, Ryan SJ, Glaser BM, Murphy RP, eds. Ocular circulation and neo-
vascularisation. Dordrecht: Martinus NijhoffDr $W$ Junk, vascularisation. Dor

25 Quinn GE, Dobson V, Barr CC, et al. Visual acuity in infants after vitrectomy for severe retinopathy of prematurity. Ophthalmology 1991;98:5-13.

26 Palmer EA, Biglan AW, Hardy RJ. Retinal ablative therapy for active retinopathy of prematurity: history, current status and prospects. In: Silverman WA, Flynn JT, eds. Contemporary issues in fetal medicine and neurology 2 . Retinopathy of prematurity. Boston: Blackwell Scientific Publications, 1985:207-28.

27 Spencer $\mathrm{R}$. The CRYO-ROP study: a national cooperative study of retinopathy of prematu JW, Mamelok AE, Mittl RN, Orellana J, eds. Treatment of retinopathy of prematurity. Chicago: Year Book Medical retinopathy of prematurity.

28 Fielder AR. Cryotherapy of retinopathy of prematurity. In Davidson SI, Jay B, eds. Recent advances in ophthalmology Davidson SI, Jay B, eds. Recent advances in ophthal

29 McNamara JA, Tasman W, Brown GC, Federman JL Laser photocoagulation for stage $3+$ retinopathy of prematurity. Ophthalmology 1991;98:576-80.

30 Landers MB, Toth CA, Semple C, Morse LS. Treatment of retinopathy of prematurity with argon laser photocoagulation. Arch Ophthalmol 1992;110:44-7.

31 Brown GC, Tasman WS, Naidoff M, Schaffer DB, Quinn
G, Bhutani VK. Systemic complications associated with retinal cryoablation for retinopathy of prematurity. Ophthalmology 1989;97:855-8.

32 Patz A. New role for the ophthalmologist in prevention of retrolental fibroplasia. Arch Ophthalmol 1967;78:565-8.

33 Cantolino SJ, O'Grady GE, Herrera JA, Israel C, Justice J, Flynn JT. Ophthalmoscopic monitoring of oxygen therapy in premature infants. Fluorescein angiography in 322-31.

34 Palmer EA. Optimal timing of examination for acute retrolental fibroplasia. Ophthalmology 1981;88:662-8.

35 Brown DR, Biglan AW, Stretavsky M. Screening criteria for the detection of retinopathy of prematurity in patients for the detection of retinopathy of prematurity in patients in a neonatal intensive care

36 Tan KEWP, Cats BP. Timely incidence of examination for acute retinopathy of prematurity (ROP) and its consequence for the screening strategy. Am $\mathcal{F}$ Perinatal 1989;6: 337-40.

37 Urrea PT, Rosenbaum AT. Retinopathy of prematurity: an ophthalmologist's perspective. In: Isenberg SJ, ed. The eye in infancy. Chicago: Year Book Medical Publishers, 1989:428-56.

38 Biglan AW, Cheng KP, Brown DR. Update on retinopathy of prematurity. Int Ophthalmol Clin 1989;29:1-9.

39 Schaffer DB. Update on retinopathy of prematurity: the examination guidelines. Seminars in Ophthalmology 1990;5:100-6.

40 Fledelius HC, Rosenberg T. Retinopathy of prematurity. Where to set the screening limits? Recommendations Where to set the screening limits? Recommendations based on two

41 Darlow BA, Clemett RS. Retinopathy of prematurity: screening and optimal use of the ophthalmologist's time. Aust NZ F Ophthalmol 1990;18:41-6. 